\title{
A Novel Technique for Measuring the Pulsewidth of Nanosecond Laser Pulses
}

\author{
Siu-Chung Tam, Member, IEEE, Wah-Peng Neo, Hock-Chuan Chua, Anthony T. S. Ho, Senior Member, IEEE, \\ and Sing Lee, Member, IEEE
}

\begin{abstract}
This paper presents a simple, low-cost, and novel technique for measuring the temporal pulse shape of a nanosecond laser pulse accurately and consistently. The measurement configuration consists of a focusing lens, a tapered rod, and a digital noise filter. Using this setup, the pulsewidth of a nitrogen laser has been determined experimentally. Compared with an unaided measurement configuration, the new setup reduces the maximum deviation from $24.5 \%$ to $6.2 \%$ and the standard deviation from $7.6 \%$ to $1.4 \%$, respectively.
\end{abstract}

Index Terms -Laser beams, laser measurements, laser stability, measurement, pulse measurements, pulsed lasers.

\section{INTRODUCTION}

$\mathbf{I}$ T IS IMPORTANT to measure accurately and consistently the pulsewidths of pulsed nanosecond lasers such as excimer lasers, the nitrogen laser, and the Q-switched Nd:YAG laser. For example, in excimer laser keratectomy, the control of the laser duration is important in determining the amount of ablation of the cornea tissues [1]. Similarly, the temporal characteristics of the laser pulse are vital in applications such as shadowgraphy, schlieren photography, microlithography, and micromachining [2].

In the literature, most researchers have reported on the use of a combination of a fast photodiode coupled to a storage oscilloscope (or a transient-event digitizer) for the measurement of pulsewidths [3], [4]. Occasionally, an expensive streak camera may be employed [5].

Generally speaking, the pulsewidth of a laser depends on the pumping configuration and the resonator parameters. For example, in the case of a nitrogen laser, the temporal pulse shape depends largely on the voltage of the discharge circuit and the pressure of the lasing gas. However, it is very difficult to obtain an accurate and consistent measurement of the pulse shape of a nanosecond laser pulse because of the following.

1) The laser pulse is of a short duration, and the pulse profile may vary from one shot to another. Therefore, to capture the pulse shape accurately, a precision transientevent digitizer or digital oscilloscope with very high sampling rate, in the range of gigasamples per second or higher, is required to capture the laser pulse shape reliably.

Manuscript received February 27, 1998; revised Decmber 21, 1998.

S.-C. Tam, W.-P. Neo, H.-C. Chua, and A. T. S. Ho are with the School of Electrical and Electronic Engineering, Nanyang Technological University, Singapore 639798.

S. Lee is with the School of Science, National Institute of Education, Nanyang Technological University, Singapore 259756.

Publisher Item Identifier S 0018-9456(98)09935-5.
2) Owing to the short pulse duration, a miniature photodiode has to be used for pulse detection. Otherwise, the carrier transit time will become very long and degrade the temporal measurements [6].

3) The spatial energy distribution of a nitrogen or excimer laser beam is generally non-Gaussian and nonuniform. Consequently, for accurate and consistent measurement of the temporal pulse shape, the laser beam has to be focused neatly onto the tiny photodiode to even out the effects of different parts of the beam. Proper alignment of the focused laser beam and the photodetector is, thus, crucial.

In this paper, we report on a new technique for the consistent measurement of laser pulsewidths. The results are compared to those obtained using a few other methods.

\section{EXPERIMENTAL LAYOUT}

The pulsed laser used in our experiment is a nitrogen laser developed in our laboratory (Blumlein-circuit, transverse flow, electrically excited, corona preionization, $2.8-\mathrm{mm}$ electrode gap) [2]. As the radiative lifetime of nitrogen's transition from the $C^{3} \pi_{\mu}$ upper lasing state to the $B^{3} \pi_{g}$ lower lasing state varies from about $40 \mathrm{~ns}$ at a few mbars to about $2 \mathrm{~ns}$ at one bar [7], fast high-voltage electrical discharges are required to excite the nitrogen molecules. Part of the kinetic energy of an electron is transferred to a nitrogen molecule by elastic collision

$$
N_{2}+e \rightarrow N_{2}^{*}+e
$$

Fig. 1 shows a schematic diagram of the experimental layout. Through numerical control, a PC is used to vary the operating voltage from 9.0 to $11.1 \mathrm{kV}$, and the gas operating pressure from 250 to 1000 mbar. Laser emission at the wavelength of $337.1 \mathrm{~nm}$ is confirmed using an Oriel 77400 spectrograph with $0.2-\mathrm{nm}$ resolution.

The output beam from the $N_{2}$ laser is split into two paths for simultaneous measurements of pulsewidth and pulse energy. The uncoated wedge reflects about $4 \%$ of the laser beam to the photodiode (MG13DAH001 with a detection area of 0.04 $\mathrm{mm}^{2}$ and a rise time of $0.35 \mathrm{~ns}$ ). A HP54720D transient-event oscilloscope, sampling at $8 \mathrm{GSa} / \mathrm{s}$ and having a rise time of $0.175 \mathrm{~ns}$, is used to record the laser pulses and, subsequently, download the data to the PC via the built-in HPIB interface.

The HP scope also provides an optional digital noise filter for the incoming signals. The digital low-pass filter, called the finite impulse-response (FIR) filter, will attenuate the 


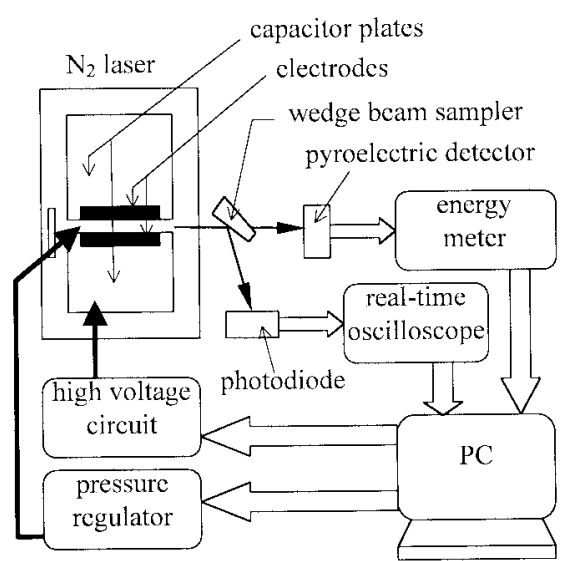

Fig. 1. Schematic diagram of experimental layout.

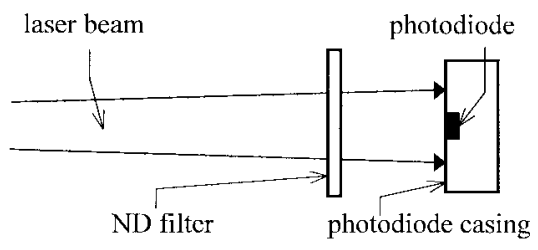

Fig. 2. Direct unaided method for pulsewidth measurement.

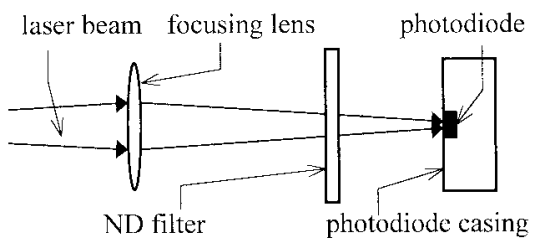

Fig. 3. Pulsewidth measurement with a focusing lens.

high-frequency components with an approximate Gaussian response. This effectively reduces both the noise floor in the scope and any noise spikes riding on the input signal, although the effective signal bandwidth is narrowed from $2 \mathrm{GHz}$ to 400 $\mathrm{MHz}$ [8].

\section{Measurement Configurations}

Fig. 2 shows the setup for direct unaided measurement of the laser pulse. The laser pulse is shone directly onto the photodiode after passing through a neutral-density (ND) filter that attenuates the intensity level of the signal to a suitable level for photodetection.

Fig. 3 shows an improved measurement setup that makes use of an equiconvex focusing lens made of fused silica. It has been found that the alignment of the photodetector with respect to the focused beam is very critical.

Fig. 4 shows the measurement setup that makes use of a 1-m-long multimode optical fiber as the beam homogenizer. It can be observed that the ND filter is no longer required, as the beam intensity at the photodetector can be adjusted by varying slightly the distance between the tip of the fiber and the photodetector.

Fig. 5 shows the novel measurement setup that makes use of a focusing lens in conjunction with a tapered rod acting as a

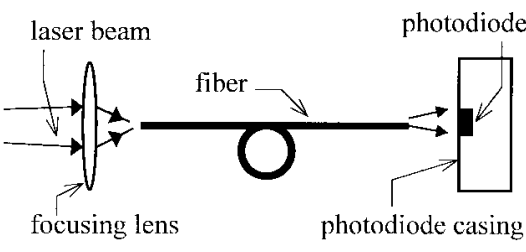

Fig. 4. Pulsewidth measurement with an optical fiber.

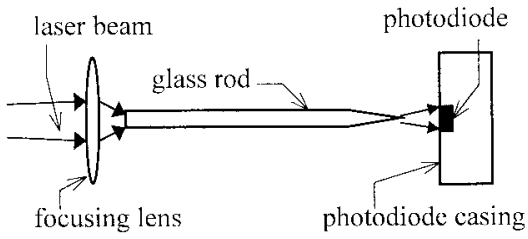

Fig. 5. Pulsewidth measurement with a tapered glass rod.

TABLE I

Pulsewidths Obtained Using Various Measurement Setups

\begin{tabular}{l|c|c|c}
\hline \multirow{2}{*}{ Measurement Setup } & \multicolumn{3}{|c}{ Pulse width of 20 pulses } \\
\cline { 2 - 4 } & $\begin{array}{c}\text { Mean } \\
\text { (nsec) }\end{array}$ & $\begin{array}{c}\text { Maximum } \\
\text { Deviation (\%) }\end{array}$ & $\begin{array}{c}\text { Standard } \\
\text { Deviation (\%) }\end{array}$ \\
\hline Unaided Direct Method & 1.50 & 24.5 & 7.6 \\
\hline $\begin{array}{l}\text { Unaided Direct Method } \\
\text { with Digital Noisc Filtcring }\end{array}$ & 1.64 & 14.1 & 3.9 \\
\hline $\begin{array}{l}\text { Focusing Lens with Digital } \\
\text { Noise Filtering }\end{array}$ & 1.73 & 9.8 & 2.9 \\
\hline $\begin{array}{l}\text { Optical Fiber with Digital } \\
\text { Noise Filtering }\end{array}$ & 1.89 & 7.6 & 2.0 \\
\hline $\begin{array}{l}\text { Tapered Glass-Rod with } \\
\text { Digital Noise Filtcring }\end{array}$ & 1.70 & 6.2 & 1.4 \\
\hline
\end{tabular}

beam homogenizer and a light pipe. The tapered rod was pulled over a flame in the laboratory. The taper angle is roughly $5^{\circ}$. Again, no ND filter is required, as the beam intensity at the photodetector is adjusted by varying the distance between the tip of the tapered rod and the photodetector.

\section{RESULTS AND DISCUSSIONS}

Table I summarizes the results of pulsewidth measurement using the various techniques: 1) the direct unaided method; 2) the direct unaided method with digital noise filtering; 3) the focusing lens method with digital noise filtering; 4) the optical fiber method with digital noise filtering; and 5) the tapered rod method with digital noise filtering. Twenty laser pulses were recorded for each setup at a discharge voltage of $11.1 \mathrm{kV}$ and a nitrogen pressure of 1 bar. The mean, maximum deviation (defined as the difference between the maximum and minimum values normalized by the mean), and standard deviation are calculated for each set of readings.

In the direct unaided method, the photodetector is sampling the laser beam directly from the exit port of the nitrogen laser. As shown in Table I, the average pulsewidth is $1.50 \mathrm{~ns}$, the maximum deviation is $24.5 \%$, and the standard deviation is $7.6 \%$. The average pulse energy, detected by a Molectron EM500 energy meter with a J-25 pyroelectric probe, is 


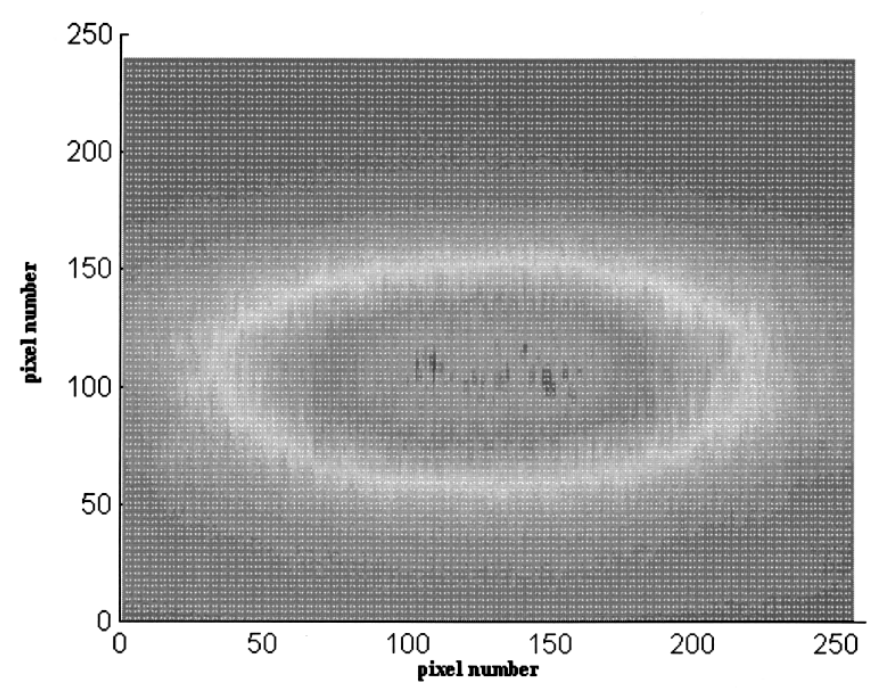

Fig. 6. Intensity profile of raw beam (discharge voltage $=11.1 \mathrm{kV}$, nitrogen pressure $=1$ bar).

typically $0.23 \mathrm{~mJ}$. The divergence of the beam is estimated to be $2^{\circ}$ approximately.

The mode shape, i.e., the transverse intensity distribution of the laser beam, is assessed using a Spiricon LBA-100A beam diagnostic system. The laser beam falls onto a Cohu 4810 CCD camera, and the intensities captured are analyzed and displayed on the monitor. The intensity profile can also be output in the form of a data file. Fig. 6 shows the intensity distribution of a typical nitrogen laser pulse captured by the beam profiler. It can be observed that the intensity distribution of the raw beam is nonuniform, but approximates a bivariate normal distribution. Energy spikes exist mainly at the center of the beam. Also, using the same apparatus, we have determined that the pointing instability of the nitrogen laser output, i.e., beam jitter, is approximately $0.22 \mathrm{mrad}$ (at $95 \%$ confidence interval). Therefore, the shot-to-shot variation over a small detector area of $0.04 \mathrm{~mm}^{2}$ becomes fairly substantial.

The digital noise filter improves pulsewidth measurement, with the maximum deviation being reduced from $24.5 \%$ to $14.1 \%$ and the standard deviation being reduced from $7.6 \%$ to $3.9 \%$. The reason is that the high-frequency noise that may exist at the peak of the pulse is filtered. The pulsewidth at full width at half maximum (FWHM) can then be determined more accurately. However, it can be observed that the mean pulsewidth is lengthened by $0.14 \mathrm{~ns}$. This is mainly caused by an increase in the rise time of the instrument by reducing the bandwidth (BW) of the oscilloscope from $2 \mathrm{GHz}$ to $400 \mathrm{MHz}$. The rise time $\left(t_{r}\right)$ is related to the $\mathrm{BW}$ by [6]

$$
t_{r}=\frac{0.35}{\mathrm{BW}}
$$

Therefore, the theoretical rise time is increased from $0.175 \mathrm{~ns}$ for $\mathrm{BW}=2 \mathrm{GHz}$ to $0.875 \mathrm{~ns}$ for $\mathrm{BW}=400 \mathrm{MHz}$.

A further experiment performed using this measurement setup reviews that the average pulsewidths for 20 pulses taken at different parts of the beam may vary by $0.20 \mathrm{~ns}$ (at $95 \%$ confidence interval) over a direct beam area of $3.5 \mathrm{~mm} \times 3.5$

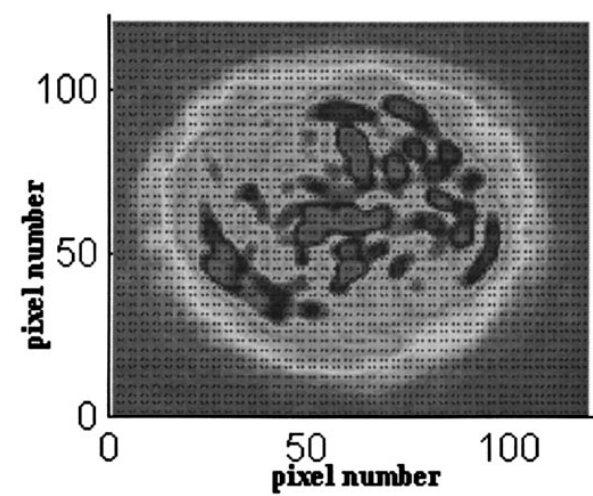

Fig. 7. Intensity profile of laser beam after exiting from optical fiber (discharge voltage $=11.1 \mathrm{kV}$, nitrogen pressure $=1 \mathrm{bar}$ ).

mm. Hence, variation of the mean pulsewidth by as much as $0.20 \mathrm{~ns}$ should not be considered as overly alarming.

Using a focusing lens with digital noise filtering improves the consistency of the measurements, with the maximum deviation being reduced to $9.8 \%$ and the standard deviation being reduced to $2.9 \%$. The improvement results from a bigger portion of the beam being captured and measured by the tiny photodetector.

Putting one end of a 1-m-long optical fiber at the focus of the converging lens allows the beam to be homogenized by mode propagation and multiple reflections in the multimode fiber. This results in the maximum deviation being reduced to $7.6 \%$ and the standard deviation being reduced to $2.0 \%$. Fig. 7 shows the intensity distribution of the laser beam emerging from the tip of the optical fiber. A large portion of the beam is homogenized, but there exist sporadic energy peaks all over the beam.

Since the optical fiber has a core diameter of $50 \mu \mathrm{m}$, a numerical aperture of 0.25 , and a refractive index of 1.46 , it works out that the modal dispersion (or the longest geometrical path difference for different ray angles in the fiber) will account for about 0.07 ns of pulse dispersion. Therefore, the increase in the mean pulsewidth is due to a combination of having temporal pulse dispersion in the fiber and having a larger portion of the beam being sampled.

It should be noted that other commercial laser beam homogenizers might be attempted to achieve better measurement consistency. These devices include "fly's eye" lenses, light ducts, and diffractive optical elements. However, such products are generally quite expensive.

The use of a tapered-end circular glass rod improves further the consistency of pulsewidth measurements. The maximum deviation and the standard deviation are reduced to $6.2 \%$ and $1.4 \%$, respectively. The glass rod acts as a beam homogenizer and light conduit, so that the effect of pointing instability is largely eliminated. Fig. 8 shows the intensity profile of the laser beam after emerging from the tapered end of the glass rod. The rays that enter the rod bounce off the sides of the glass wall for at least four times, thus homogenizing the beam to a certain extent. In this setup, since the numerical aperture of the input beam is about 0.18 , the increase in average pulsewidth due to beam propagation in the tapered glass rod is only about 0.006 ns. 


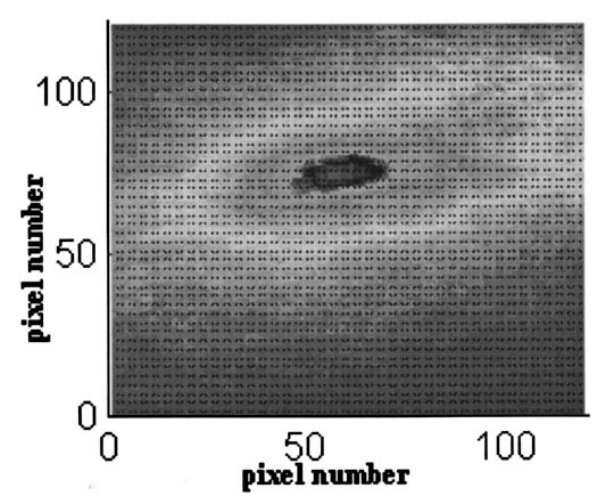

Fig. 8. Intensity profile of laser beam after exiting from tapered rod (discharge voltage $=11.1 \mathrm{kV}$, nitrogen pressure $=1 \mathrm{bar}$ ).

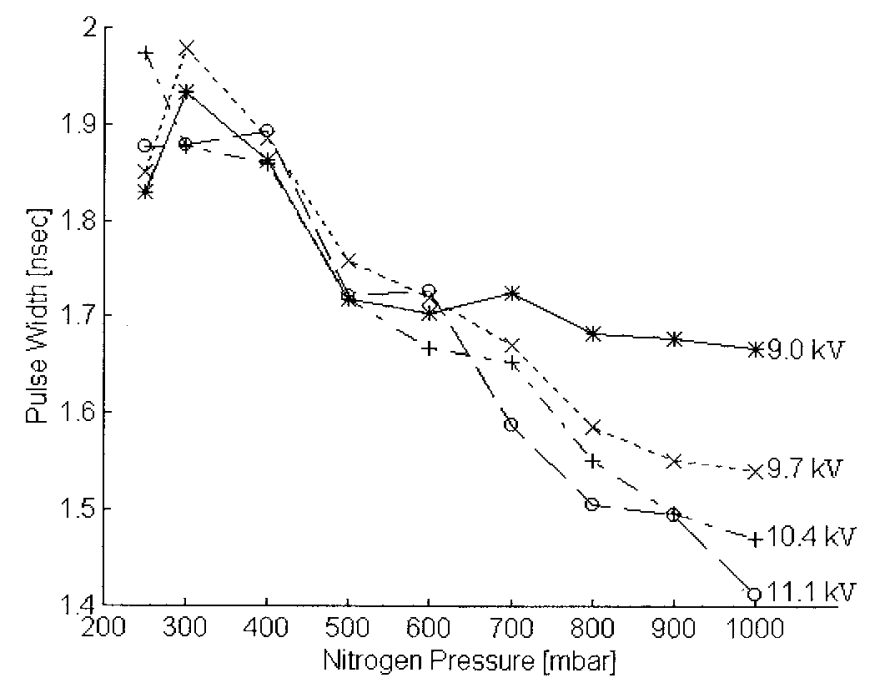

Fig. 9. Pulsewidth measurement at various discharge voltages and nitrogen pressures using the unaided direct method.

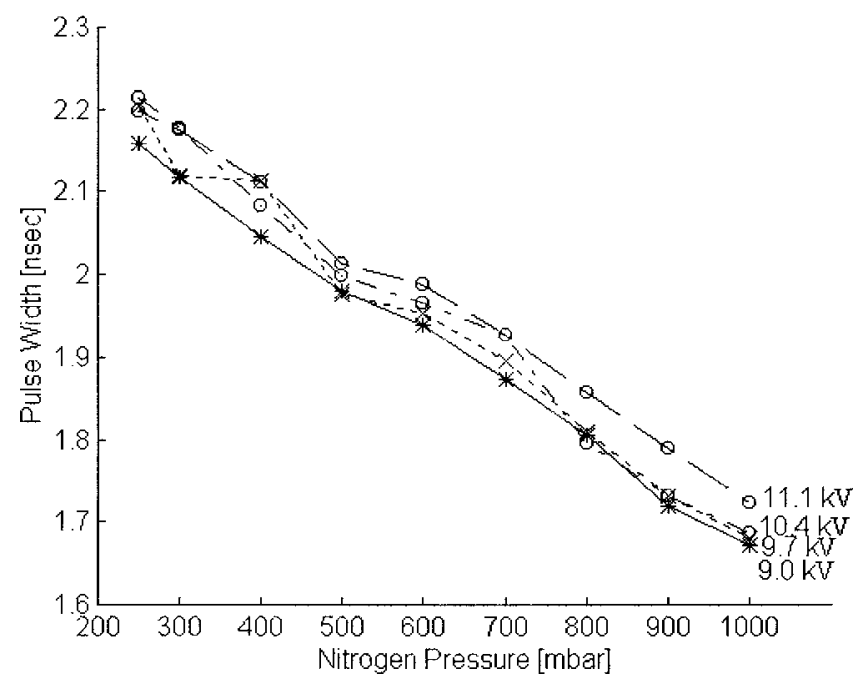

Fig. 10. Pulsewidth measurement at various discharge voltages and nitrogen pressures using the proposed tapered-rod method.

Fig. 9 shows the pulsewidth measurements using an unaided measurement setup for various nitrogen gas pressures at different discharge voltages. The same measurements were retaken using the proposed measurement method (focusing lens + tapered glass rod + digital noise filter), and the results are plotted in Fig. 10. The improvement in data reliability and data interpretation is evident. Such improvement in measurement consistency is essential in designing hardware or software control schemes to stabilize the output of a nanosecond laser.

\section{CONCLUSION}

This paper has presented a novel, low-cost, simple, and practical technique for measuring the pulsewidth of a nanosecond pulsed laser beam. Compared with an unaided measurement setup, the use of our proposed measurement configuration (focusing lens + tapered rod + digital noise filter) improves markedly the reliability of the data collected. In our experiment with a nitrogen laser, the maximum deviation has been reduced from $24.5 \%$ to $6.2 \%$, and the standard deviation from $7.4 \%$ to $1.4 \%$. This is achieved by partial homogenization of the raw beam, and by elimination of the adverse effect of beam jitter. The lengthening of the pulse due to modal dispersion is negligible.

\section{REFERENCES}

[1] G. H. Pettit, M. N. Ediger, and R. P. Weiblinger, "Excimer laser ablation of the cornea," Opt. Eng., vol. 34, no. 3, pp. 661-667, 1995.

[2] S. Lee, S. Kumar, T. C. Chia, A. Serban, and S. V. Springham, "Pulse charging of capacitors for reduction of voltage stress," Rev. Sci. Instrum., vol. 65 , no. 7, pp. 2408-2409, 1994.

[3] H. M. von Bergmann, "Sealed-off, miniature, high-power nitrogen laser," J. Phys. E, Sci. Instrum., vol. 10, pp. 1210-1212, 1977.

[4] A. Hariri, M. Tarkashvand, and A. Karami, "Corona-preionized nitrogen laser with variable pulse width," Rev. Sci. Instrum., vol. 61, no. 5, pp. 1408-1412, 1990.

[5] M. Kovach, private communication, 1998.

[6] K. D. Li Dessau, "Application note 1: Insights into high-speed detectors and high-frequency techniques," New Focus Inc., Santa Clara, CA, 1991.

[7] S. V. Rodrigues and W. Baumann, "A small rugged nitrogen laser for instrumentation," Rev. Sci. Instrum., vol. 64, no. 1, pp. 63-70, 1993.

[8] User's Reference for HP54720D, Hewlett-Packard Company, Palo Alto, CA, 1995, pp. 3-6.

Siu-Chung Tam (M'93), photograph and biography not available at the time of publication.

Wah-Peng Neo, photograph and biography not available at the time of publication.

Hock-Chuan Chua, photograph and biography not available at the time of publication.

Anthony T. S. Ho (M'90-SM'94), photograph and biography not available at the time of publication.

Sing Lee (M'96), photograph and biography not available at the time of publication. 